\title{
Moodle como soporte de la gestión de contenidos educativos en universidades cubanas desde la perspectiva del proyecto ELINF
}

\section{Moodle as Support for the Management of Educational Contents in Cuban Universities From the Perspective of the ELINF Project}

\author{
DrC. Carlos Rafael Batista Matamoros_m carlosb@,uho.educ.cu $\square$ https://orcid.org/0000-0001-5210-7721 \\ Lic. Lourdes María Campins Marrero_w lcampins@uho.edu.cu_m https://orcid.org/0000-0003-0739-8405 \\ DrC. Ronal Tamayo Cuenca_m ronaltc@uho.edu.cu_wttps://orcid.org/0000-0001-5210-7721 \\ Universidad de Holguín, Cuba.
}

Fecha de recepción: 22 de abril de 2020

Fecha de aceptación: 1 de mayo de 2020

Fecha de publicación: 30 de junio de 2020

Favor de citar este artículo de la siguiente forma:

Batista Matamoros, C., Campins Marrero, L., y Tamayo Cuenca, R. (2020). Moodle como soporte de la gestión de contenidos educativos en universidades cubanas desde la perspectiva del proyecto ELINF.

AULA, revista de Humanidades y Ciencias Sociales, 66 (2), 17-25.

DOI:https: //doi.org/10.33413/aulahcs.2020.66i2.133

\section{RESUMEN}

La presente investigación tiene como objetivo establecer valoraciones críticas sobre factores relativos a la gestión de contenidos educativos ante los modelos pedagógicos del siglo XXI, caracterizados por el uso extensivo e intensivo de las Tecnologías de la Información y las Comunicaciones (TIC); se analiza su operacionalización mediante los sistemas para la gestión del aprendizaje, en general y Moodle en particular. Se exponen los elementos que estimulan o constituyen barreras para la aplicación de la plataforma en el contexto de la educación superior cubana. Se muestran los aportes y desarrollos basados en la visión distintiva del proyecto ELINF ${ }^{1}$ sobre el uso de Moodle en universidades cubanas, que destaca no solo por las bondades que se obtienen a partir de su gestión sino de las que se podrían lograr a través de la interoperabilidad de los metadatos de la plataforma con los de otros sistemas para el aprendizaje y la investigación existentes tanto en el ecosistema de aplicaciones del propio proyecto, como de la educación superior cubana en general. Esto genera la posibilidad de brindar innumerables servicios y funcionalidades de apoyo a los procesos de investigación, enseñanza y aprendizaje en los ámbitos del pregrado, postgrado y la educación con un enfoque abierto; elementos que contribuyen con el objetivo cuatro de Agenda 2030 para el Desarrollo Sostenible establecida por la ONU.

Palabras clave: TIC, sistemas para la gestión del aprendizaje, Moodle, interoperabilidad, educación superior, educación abierta, aprendizaje, investigación.

${ }^{1}$ ICT Supporting the educational processes and the knowledge management in higher education» cómo parte del Programa de Red de Cooperación Interuniversitaria cubano-flamenca REDTIC «Strengthening of the role of ICT in Cuban Universities for the development of the society. 


\section{ABSTRACT}

The present investigation aims to establish critical assessments of factors related to the management of educational content in the face of the pedagogical models of the 21 st century characterized by the extensive and intensive use of Information and Communication Technologies (ICT); analyzes its operationalization through systems for learning management in general and Moodle in particular. The elements that stimulate or are barriers to the application of the platform in the context of Cuban higher education are exposed. The contributions and developments are shown from the distinctive vision of the ELINF project, about the use of Moodle in the Cuban university, that stands out not only for the benefits that can be obtained from its management, but from those that can be achieved through the interoperability of the metadata of the platform with systems for learning and research in both the application ecosystem of the project itself and Cuban higher education in general; This creates the possibility of providing innumerable services and functionalities to support the research, teaching and learning processes both in the undergraduate and postgraduate fields, providing a framework for open education; elements that supports the objective four of the 2030 Agenda for Sustainable Development established by the UN.

Keywords: ICT, systems for learning management, Moodle, interoperability, higher education, open education, learning, research.

\section{Introducción}

Los modelos pedagógicos del siglo XXI se desarrollan en un entorno basado en el uso extensivo e intensivo de las Tecnologías de la Información y las Comunicaciones (TIC), que estimulan un cambio de perspectiva en las propias dinámicas organizacionales de generar, intercambiar y asimilar conocimiento (Franco y Pinho, 2019). Así también ocurre en el ámbito docente, donde en períodos previos el encuentro presencial constituye el eje sobre el que giraba el proceso de enseñanza-aprendizaje, contexto en el cual el profesor se erige como ente central que resume y distribuye el conocimiento hacia el grupo de educandos.

Sin embargo, las innovaciones en el campo de las tecnologías de la información y la comunicación, así como, su aplicación intensiva a ámbitos de la vida social, política, económica y su impacto en la vida diaria de millones de seres humanos; propicia un ambiente donde los espacios de intercambio de información y conocimiento confluyen muchas veces a través de dispositivos tecnológicos como las plataformas digitales. En este contexto, el ser humano se encuentra dotado de posibilidades y herramientas que permiten un amplio acceso a la información a través de espacios de colaboración con expertos o aprendices en un campo determinado, ambientes personalizados y una mayor independencia en el proceso de generación, intercambio y apropiación de conocimientos.

Los elementos anteriores generan nuevos modelos pedagógicos que mediante un enfoque socio-constructivista, objetivan potenciar factores como:

-La habilidad e independencia cognoscitiva: Entendida «desde la perspectiva del aprendizaje autónomo, el manejo de las estrategias cognitivas y metacognitivas de aprendizaje se convierten en un elemento clave que permite al estudiante orientarse en la información disponible mediante su organización, clasificación e interpretación, así como la organización, supervisión y evaluación del propio proceso de aprendizaje». (Klimenko, 2009).

-La interacción profesor-estudiante: esta relación mantiene los mismos principios establecidos por los modelos pedagógicos tradicionales, dichos principios no cambian con el uso e implementación de las TIC, solo se encuentran condicionados por el tiempo y el espacio, pero 
siempre, «una buena guía del maestro sobre el alumno resulta alentador para el estudiante y su rendimiento académico, ya que la motivación e incluso la cercanía de los alumnos con los maestros depende en gran medida del grado de identificación que los alumnos tengan hacia éstos, así como el que estos últimos (usen) técnicas de enseñanza o métodos pedagógicos, que sepan despertar en los alumnos el interés». (Llanga, Murillo, Panchi, Paucar y Quintanilla, 2019).

-La interacción estudiante-estudiante: los estudiantes se convierten en agentes activos de intercambio de información, así como de la creación de nuevos contenidos y conocimiento. En este contexto se potencia de manera orgánica la creatividad al combinar el proceso docente y educativo con factores lúdicos y de actualidad como las redes de colaboración y sociales.

-La motivación, que Llanga, et al., (2019) plantean: «desde un punto etimológico es la combinación de dos palabras, motivo y acción. Por lo mismo se entiende que, es el motivo o razón que se toma para realizar ciertas acciones con el fin de llegar a una meta. Se conoce a esta como un motor que todas las personas poseen ya que es parte de la naturaleza del ser humano. En el inicio de todo... el proceso de aprendizaje, además de poder atender, es necesario e importante querer aprender, esto involucra una cierta motivación inicial».

A su vez, la motivación académica posee tres componentes básicos, que Núñez identifica como: "Componentes de valor, de expectativa y afectivo que, respectivamente se refieren a los motivos y razones para la realización de una actividad en donde la persona le asigna un grado de importancia a la actividad y se fijan metas de aprendizaje; a que se da a conocer una crítica individual sobre la capacidad de ejecutar una actividad, es decir, el mismo individuo mide sus posibilidades para realizar con éxito la actividad, y el que se relaciona con los sentimientos y emociones que se producen al realizar una actividad, en este caso depende del interés del individuo de realizar dicha actividad» (Núñez, 2009), por lo que es posible aseverar que la motivación académica está vinculada con el proceso cognitivo del estudiante que le permite lograr sus metas.

-La ubicuidad de la clase, donde Caldeiro (2015) reconoce que «el aprendizaje ubicuo es aquel que se produce en contextos diversos que trascienden el escenario delimitado por el espacio tiempo de una clase tradicional» y a su vez señala que, «esta modalidad puede ser experimentada en diferentes grados por lo que podemos reconocer, entre las prácticas de enseñanza, tres niveles de ubicuidad: a) actividades delimitadas espacial y temporalmente dentro del salón de clases, b) entornos educativos en línea que extienden el aula pero que están fuertemente controlados por el docente, emulando las dinámicas de las aulas presenciales y c) la utilización de espacios públicos digitales como contextos en los cuales pueden proponerse actividades didácticas a través de la resignificación pedagógica». (Caldeiro, 2015).

-Innovación en la docencia: las investigadoras Margalef y Arenas (2006) exponen que, «cuando hablamos de innovación educativa aparecen fuertemente entremezcladas las nociones de cambio y reforma; donde el concepto de innovación educativa lo entendemos a partir de las siguientes características:

- Supone una idea percibida como novedosa por alguien, y a su vez incluye la aceptación de dicha novedad.

- Implica un cambio que busca la mejora de una práctica educativa.

- Es un esfuerzo deliberado y planificado encaminado a la mejora cualitativa de los procesos educativos.

- Conlleva un aprendizaje para quienes se implican activamente en el proceso de innovación.

- Está relacionado con intereses económicos, sociales e ideológicos que influyen en todo proceso de innovación».

-Innovación abierta: «considera las fuentes de conocimiento a través de asociaciones 
MOODLE COMO SOPORTE DE LA GESTIÓN

DE CONTENIDOS EDUCATIVOS EN UNIVERSIDADES CUBANAS

DESDE LA PERSPECTIVA DEL PROYECTO ELINF

y colaboraciones soportadas con tecnología para trabajar con los grupos de interés y otros actores de la sociedad y así propiciar la inteligencia colectiva al servicio de la innovación empresarial» (Chesbrough, 2006; Chesbrough et al., 2008; Dahlander y Gann, 2010; Enkel et al., 2009, citados por Álvarez-Aros y Bernal-Torres (2017).

-Formación en línea, según Grau-Perejoan (2008), se trata de «una modalidad de formación que contempla habitualmente un uso extensivo de las tecnologías de la información y la comunicación (TIC), que explota los diferentes recursos tecnológicos ofrecidos por Internet y que tiene como principales características distintivas el hecho de que se desarrolla en un espacio virtual y que la comunicación entre los participantes de las actividades se realiza a través de este espacio y/o a través de e-mail». Se distingue entre sus variadas ventajas por su extensión, ya que facilita promover y realizar cualquier acción formativa a diferentes lugares o países, permitiendo el registro de todas las actividades realizadas, tanto por profesores, como estudiantes, en los espacios virtuales de almacenamiento, sumado a que permite ahorrar tiempo y costos de viajes. Es necesario reconocer que tiene como inconvenientes la necesaria inversión que se debe realizar, tanto en los medios tecnológicos, como en los recursos humanos requeridos para gestionar los mismos, así como las dificultades que pueden presentar los estudiantes, ya sea porque este tipo de formación exige autodisciplina, generarles sensación de aislamiento al trabajar en soledad, o tener limitaciones tecnológicas que incidan en su motivación académica.

-Educación abierta: la iniciativa OpenEd Framework de la Unión Europea define como la «forma de llevar a cabo la educación que frecuentemente utiliza tecnologías digitales». $\mathrm{Su}$ objetivo es ampliar el acceso y la participación de todos, eliminando las barreras y haciendo que el aprendizaje sea accesible, abundante
AULA, Revista de Humanidades y Ciencias Sociales

y personalizable para todos. Ofrece múltiples formas de enseñar y aprender, construir y compartir conocimiento, así como una variedad de rutas de acceso a la educación formal y no formal, articulándolas de manera eficaz.

-Gestión de entornos virtuales de aprendizaje: cuando se gestiona un recurso de cualquier índole se tienen presente cada uno de los detalles que componen la organización y el recurso a representar. Salinas (2015) plantea que en cuanto a los sistemas de gestión de entornos virtuales de aprendizaje "se distinguen tres niveles distintos de decisiones en cuanto al diseño y desarrollo de las posibilidades de los entornos virtuales de formación y estos tres niveles van a dar forzosamente lugar a tres niveles o tipos de gestión de los mismos:

1. Estrategias de introducción y/o implementación, que hacen referencia a la gestión de los procesos de política institucional, de análisis del contexto, de implementación, dirigidos a la definición y puesta en marcha de un proyecto de e-learning o de utilización de TIC.

2. Estrategias de implantación y diseminación en la institución. En este nivel situamos la gestión del entorno virtual que hace referencia al proceso de convertir el e-learning en parte de la cultural de la institución.

3. De práctica y experiencia diaria dirigida a escoger la más adecuada combinación de métodos, medios y técnicas que ayude al alumno a alcanzar la meta deseada del modo más sencillo y eficaz. En otras palabras, diseñar y ejecutar estrategias didácticas». Salinas (2015).

Para hacer operacionles los cambios y adaptaciones a nuevos contextos en los modelos pedagógicos que necesitan de entornos virtuales de aprendizaje, surgen los Learning Management Systems más conocidos como LMS o Sistemas para la Gestión del Aprendizaje. Estos permiten ampliar la docencia en los ámbitos de pre y post grado en las universidades, incluso fuera de las fronteras de la institución educativa, a través de flujos de enseñanza y control del aprendizaje sincrónicos o asincrónicos. 
Los Ambientes Virtuales de Enseñanza Aprendizaje (AVEA) son considerados como:

- «Plataformas para organizar, en el tiempo y en el espacio, elementos como el contenido, la interacción y la evaluación» (Barbosa, 2006).

- «Programas informáticos interactivos de carácter pedagógico» (FEDE, 2010).

- «Escenarios tecnológicos y de servicios interconectados para contextos educativos» (Ciudad, 2016).

En estas definiciones se observa una tendencia a resaltar la importancia del vínculo entre lo pedagógico y lo tecnológico para desarrollar un proceso de enseñanza aprendizaje con interactividad y comunicación sincrónicas o asincrónicas.

En la actualidad existe una gran variedad de AVEA, entre los que destaca MOODLE, el cual, según la firma consultora docente Teachthought, resalta entre los quince mejores de su tipo a el nivel internacional en cuanto a indicadores clave como la gestión de objetos y recursos de aprendizaje, el soporte a fórums y weblogs, la capacidad de ofrecer videoconferencias y la posibilidad de disponer de recursos para la gamificación del aprendizaje, entre otros.

El sistema Moodle cuenta con una curva de experiencia de desarrollo de 19 años, encontrándose en su versión 3.7. En la actualidad se haya desplegado en 231 países (Computer Desktop Encyclopedia) y cumple con los estándares de calidad y software libre para plataformas de su tipo, lo que le otorga una amplia capacidad de respuesta a necesidades de instituciones educativas, docentes e investigadores; así como interoperabilidad entre plataformas digitales diversas en los ecosistemas de educación superior.

En el escenario cubano, Moodle constituye una oportunidad de potenciar la educación presencial, semipresencial y a distancia en los ámbitos de pregrado y postgrado, así como para contribuir con la Agenda 2030 para el Desarro1lo Sostenible adoptada por la ONU en su objetivo cuatro, que se refiere a «Garantizar una educación inclusiva, equitativa y de calidad y promover oportunidades de aprendizaje durante toda la vida para todos»».

\section{El uso del Moodle en el contexto}

de la educación superior cubana: logros, retos, interoperabilidad y posibilidades desde el proyecto ELINF

Analizando el término interoperabilidad, El-Diraby, Krijnen \& Papagelis (2017) la definen como «la capacidad de dos o más componentes de software para interactuar y comunicarse de manera significativa a través del intercambio de datos»». Lograr esta capacidad en plataformas del ámbito universitario, ofrece una gama diferente de servicios que potencian la integración de los sistemas de gestión bibliográfica (ABCD), repositorios (Dspace) y gestión de información de investigadores (VIVO).

Tomando como referencia el ecosistema del proyecto ELINF (Goovaerts, Ciudad \& Benítez, 2016), se requiere para la educación que exista interoperabilidad entre Moodle, Dspace, ABCD y VIVO.

En este sentido, Moodle es una plataforma para gestionar el proceso de enseñanza aprendizaje de forma virtual. En ella se encuentran diferentes contenidos que el profesor pone a disposición de los estudiantes, los cuales se rigen por una estructura mínima (Cañizares et al., 2014) asumida por el proyecto VLIR en Cuba en función de lograr:

- Un espacio de bienvenida.

- Un espacio de información general de los cursos.

- Un espacio para las unidades y temas.

Estos contenidos dependen de la intencionalidad pedagógica para el proceso de formación. Por tanto, se mezclan diferentes recursos (libros, artículos, tesis, imágenes, etc.) que pueden estar duplicados en otras plataformas como Dspace y ABCD. Para evitar esta situación, es necesario valorar donde debe ir cada recurso según las potencialidades de cada plataforma. 
Para ello, en el año 2014 se acordó que toda la producción científica de las universidades del proyecto, debía formar parte de las colecciones del repositorio institucional en DSpace. La relación entre Moodle y DSpace es muy importante para la formación de los profesionales dado que todos los resultados de investigación aportan al nuevo conocimiento. Entonces los cursos en Moodle necesitan realizar hipervínculos con las tesis y artículos que se alojan en DSpace. Esto permite que no se incremente en cantidad los recursos de un curso en Moodle y que se puedan localizar mejor los recursos de investigación.

De la misma forma, ABCD se encarga de gestionar la bibliografía existente en las bibliotecas de las universidades. La interoperabilidad de esta plataforma con Moodle permite que el estudiante no tenga que ir personalmente a realizar la búsqueda en esa institución, sino que, desde cualquier computadora donde esté gestionando su aprendizaje, puede buscar bibliografía orientada por el profesor, ya sea en formato digital o en formato de papel. Por último, todo lo que se produce por los investigadores se puede organizar en perfiles a través de VIVO. En este sentido Moodle es una herramienta que necesita incorporar estos perfiles de los profesores que gestionan los cursos y, a la misma vez sugerirle a los estudiantes otros posibles investigadores que se relacionen con las materias de estudio.

Visto de forma general para la educación (Figura 1), Moodle es el centro del proceso de enseñanza aprendizaje y en él se alojan programas, guías de estudio, bibliografías, conferencias, orientaciones para diferentes formas de organización, contenidos específicos de las materias a aprender, entre otros. DSpace constituye entonces una plataforma de apoyo con toda la producción científica de cada universidad, reconociéndola y resguardándola para interoperar con Moodle a través de hipervínculos o con accesos directos desde módulos desarrollados para interoperar. Por su parte la función principal de interoperabilidad de ABCD se centra en gestionar desde la red, toda la bibliografía existente en los catálogos de las bibliotecas mientras el estudiante aprende en Moodle. Por último, VIVO ofrece funcionalidades como los perfiles de los investigadores, los cursos que estos pueden tener en Moodle, materias relacionadas con estrategias de búsquedas en los diferentes sistemas, entre otras.
Figura 1. Relaciones de interoperabilidad entre Moodle, DSpace, ABCD y Vivo para la educación. (Elaboración propia).

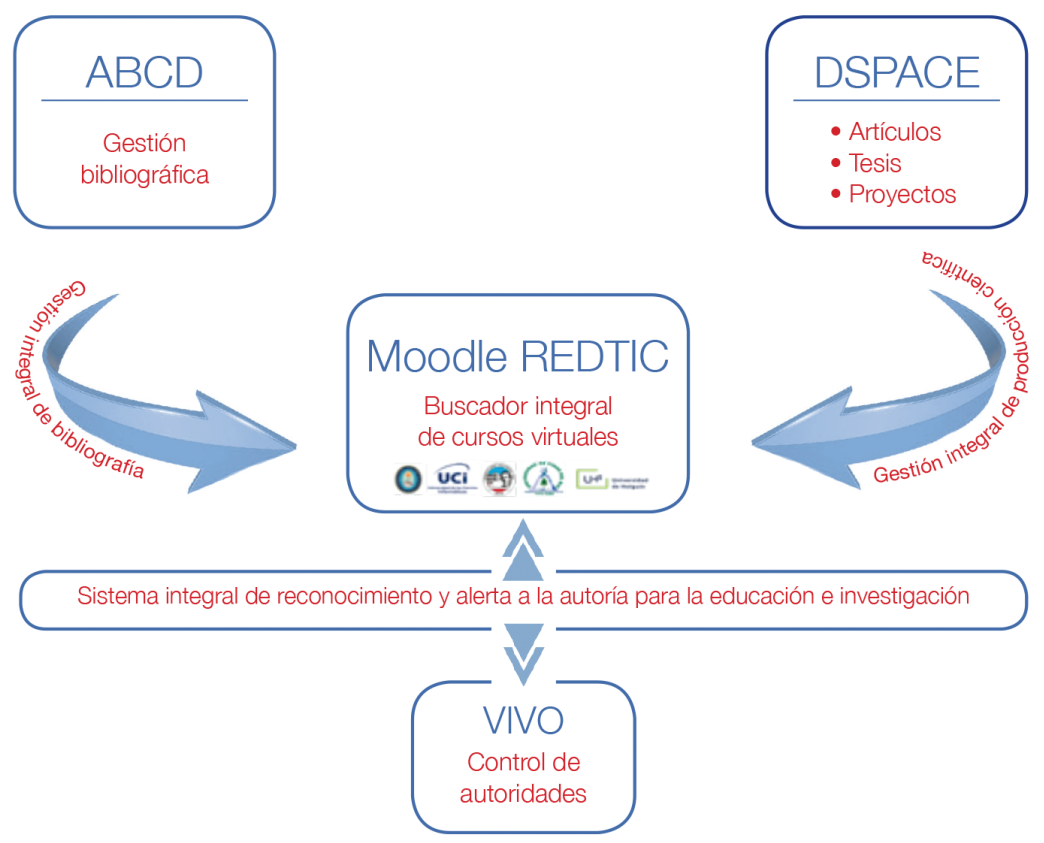




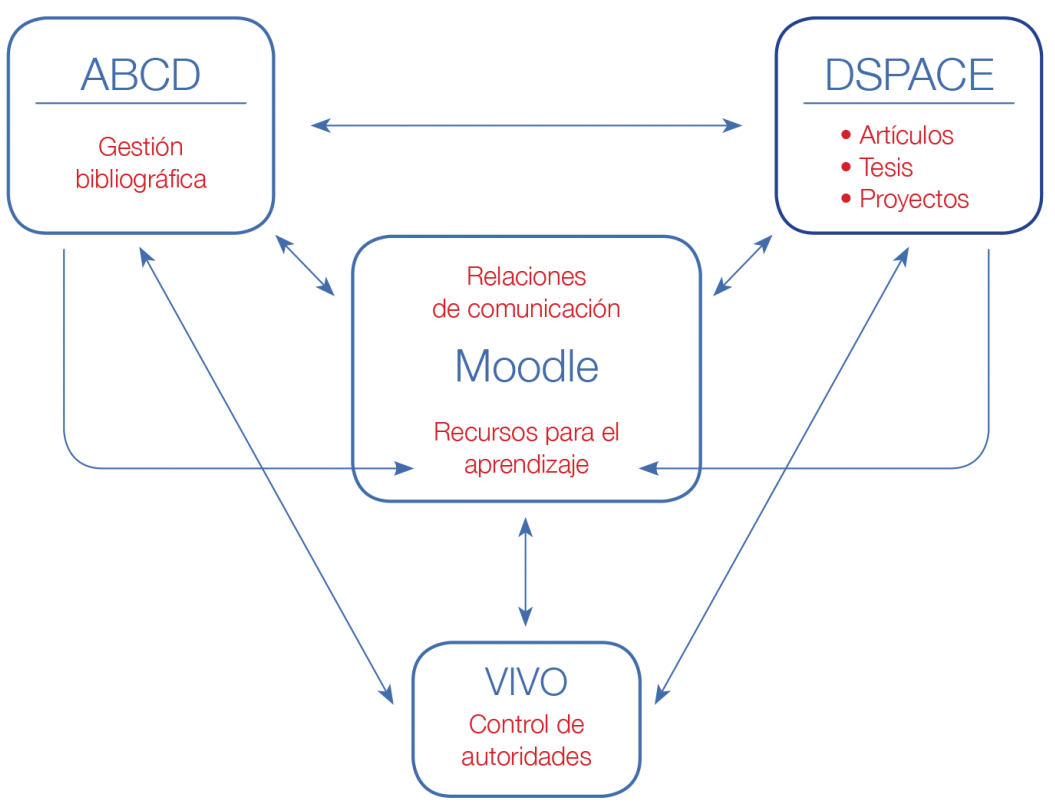

Figura 2. Relaciones de interoperabilidad entre las plataformas de las universidades miembros del proyecto ELINF. (Elaboración propia)

Además de estas relaciones, y para lograr mayor impacto, se hace necesario una mayor interoperabilidad entre las plataformas que existen en todas las universidades del proyecto. Para ello, se puede observar en la figura 2 una propuesta general de servicios integrados basados en las relaciones que se declararon anteriormente.

En la figura 2 se declara a la plataforma Moodle de REDTIC como centro de la gestión para ofrecer cursos virtuales a toda la comunidad nacional e internacional. Esta plataforma debe lograr enlazarse con todas las plataformas Moodle de las universidades del proyecto a través de un buscador de cursos, de forma tal que tanto estudiantes como profesores puedan acceder a la información que existe acerca de las asignaturas en cada universidad. Además, puede incorporarse un buscador de DSpace que enlace a todos los repositorios institucionales, lo cual permitiría tener a disposición toda la producción científica de esas universidades. De la misma forma, se lograría otro impacto con $\mathrm{ABCD}$, al gestionar integralmente toda la bibliografía existente para ofrecer a los estudiantes la disponibilidad en cada universidad.

Esta visión de interoperabilidad permitiría a nivel de país, obtener información de los elemen- tos de interés existentes en las distintas plataformas, de una manera ontológica. Por lo tanto, se abriría el diapasón de opciones para investigadores, profesores y estudiantes al ofrecer una cantidad numerosa de recursos para observar, utilizar, comparar e inclusive como materia prima para crear. En resumen, la interoperabilidad constituye una herramienta y, una cualidad que eleva la calidad de los procesos de educación e investigación en las universidades. Lograr este fin requiere de equipos especializados con altos niveles de comunicación para mantener la capacidad de intercambio entre las plataformas, independientemente de los cambios en sus versiones y tecnologías.

\section{Conclusiones}

Los modelos pedagógicos del siglo XXI están caracterizados por el uso extensivo e intensivo de las Tecnologías de la Información y la Comunicación (TIC), el desarrollo de redes y espacios de colaboración para el planteamiento y solución de problemas y cambios en los modos de asumir la docencia e investigación. Lo anterior impacta por tanto a la gestión de contenidos educativos y los modos en que esta potencia el aprendizaje con una mayor flexibilidad, a 
MOODLE COMO SOPORTE DE LA GESTIÓN

DE CONTENIDOS EDUCATIVOS EN UNIVERSIDADES CUBANAS

DESDE LA PERSPECTIVA DEL PROYECTO ELINF
AULA, Revista de Humanidades y Ciencias Sociales través de las herramientas que hoy tienen a su disposición los estudiantes en el ámbito de sociedad de la información y del conocimiento, donde existen límites como la clase presencial, el tiempo o la manera en que se crean los contenidos con enfoque abierto, social y constructivista.

En el contexto de la educación superior en Cuba, el uso de Moodle como plataforma para la gestión de la enseñanza, el aprendizaje y apoyo a la investigación, constituye una oportunidad que permite minimizar costos materiales y estimular la educación a distancia en los ámbitos de pregrado y postgrado con un enfoque abierto. Esta se constituye por tanto, en un vehículo factible para contribuir con la Agenda 2030 para el Desarrollo Sostenible adoptada por la ONU en su objetivo cuatro: Garantizar una educación inclusiva, equitativa y de calidad, y promover oportunidades de aprendizaje durante toda la vida y para todos.

\section{Referencias}

Álvarez-Aros, Erick L. y Bernal-Torres, César A. (2017). Modelo de Innovación Abierta: Énfasis en el potencial humano. Información Tecnológica. 28(1), pp.65-76. Recuperado de http://dx.doi.org/10.4067/ $\underline{\mathrm{S} 0718-07642017000100007}$

Barbosa, J. (2006). Textos publicados para el «Diplomado en Formulación de Proyectos de Virtualización» de la Pontificia Universidad Javeriana. Colombia. Recuperado de https://docplayer.es/90113523-Nombre-del-programa-diplomado-formulacion-de-proyectos-de-virtualizacion-nombre-de-la-institucion-oferente-pontificia-universidad-javeriana.html.

Caldeiro, G. (2015). Aprendizaje ubicuo. Oportunidades para el desarrollo de propuestas educativas en línea. Recuperado de https://www.researchgate.net/publication/284645999

Cañizares, R., Ruiz, L., Legañoa, M., Escalona, P y Villad, E. (2014). Estructura mínima que cumplir por los cursos a publicar en el Sistema de Gestión del Aprendizaje (Moodle). Recuperado de http://moodle.uho.edu.cu/ $\mathrm{mod} /$ resource/view.php?id=14714

Ciudad, A.F. (2016). Diseño de entornos virtuales para la integración academia-industria: implementación en la
Los avances en el despliegue de Moodle dentro de la educación superior cubana permiten pasar a una segunda fase, en la que se hace necesario ampliar las posibilidades que ofrece el sistema mediante la implementación de servicios de interoperabilidad con otras plataformas existentes en nuestro contexto, que permiten gestionar el aprendizaje y la investigación sobre todo ante necesidades del sistema educativo actual como la educación y ciencia abiertas.

El proyecto ELINF en su segunda fase, tiene como objetivo lograr la interoperabilidad entre el ecosistema de aplicaciones que el mismo ha proporcionado para potenciar la gestión de la enseñanza, el aprendizaje e investigación en la educación superior cubana. Como parte de su estrategia de trabajo desarrolla a nivel teórico, metodológico y práctico, tecnologías para lograr la interoperabilidad de los metadatos de Moodle con las restantes plataformas del proyecto e inclusive, con otros futuros desarrollos.

Disciplina Ingeniería y Gestión de Software. La Habana: Publicia. DOI: 10.4067/S0718-07642017000100007

Llanga Vargas, E.F., Murillo Pardo, J.J., Panchi Moreno, K.P., Paucar Paucar, P.P. y Quintanilla Orna, D.T. (2019). La motivación como factor en el aprendizaje. Revista Atlante: Cuadernos de Educación y Desarrollo. Recuperado de https://www.eumed.net/ rev/atlante/2019/06/motivacion-aprendizaje.html// hdl.handle.net/20.500.11763/atlante1906motivacion-aprendizaje

El-Diraby, T., Krijnen, T. \& Papagelis, M. (2017). BIM-based collaborative design and socio-technical analytics of green buildings. Automation in Construction, 82, pp.59-74. Recuperado de https://doi.org/10.1016/j.autcon.2017.06.004

FEDE. (2010). Entorno de aprendizaje virtual. Disponible en http://www.fed.ean.edu.co

Franco, M., \& Pinho, C. (2019). A case study about cooperation between University Research Centres: Knowledge transfer perspective. Journal of Innovation \& Knowledge, 4 (1). pp.62-69.

Goovaerts, M., Ciudad, F. A. y Benítez, D. (2016). Desarrollo de una red virtual de investigación y educación 
para la información científico en Cuba. Congreso Internacional de Información INFO 2016. Recuperado de http:/www.congresoinfo.cu/index.php/info/2016/ paper/viewFile/139/48.

Grau-Perejoan, O. (2008). Formación on-line. Viguera Editores. EDUC MED; 11 (3). pp.139-146.

Klimenko, O. (2009). La enseñanza de las estrategias cognitivas y metacognitivas como una vía de apoyo para el aprendizaje autónomo en los niños con déficit de atención sostenida. Revista Virtual Universidad Católica del Norte. (270). pp.1-19.

Margalef García, L. y Arenas Martija, A. (2006). ¿Qué entendemos por innovación educativa? A propósito del desarrollo curricular. Perspectiva Educacional, Formación de Profesores. (47). pp.13-31.

Núñez, J. (2009). Motivación, aprendizaje y rendimiento académico. Trabajo presentado en el X Congresso Internacional Galego-Português de Psicopedagogía. Recuperado de http://www.educacion.udc.es/grupos/ gipdae/documentos/congreso/Xcongreso/pdfs/cc/ cc3.pdf

REDTIC ICT Network Project. Recuperado de https://redtic. uclv.cu/

Salinas, J. (2015). La gestión de los entornos virtuales de formación. Recuperado de DOI: 10.13140/ RG.2.1.4634.5041
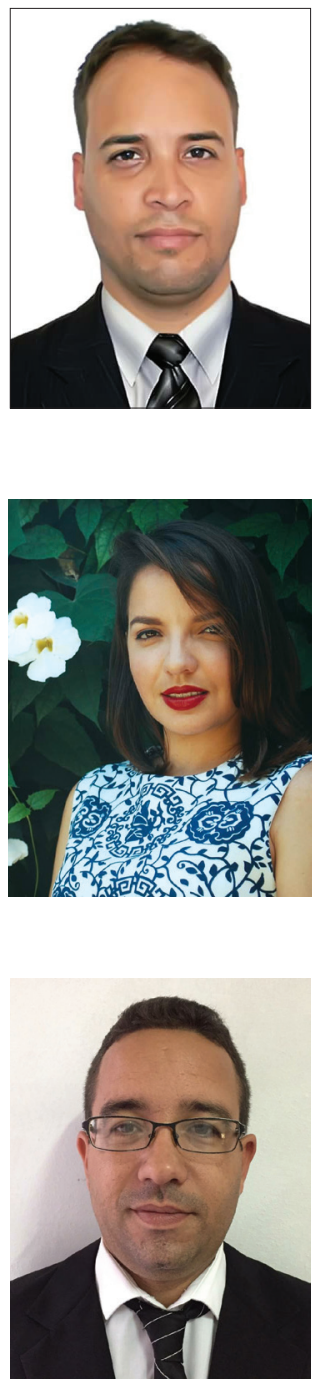

\section{DrC. Carlos Rafael Batista Matamoros}

Director de Información Científico Técnica. Universidad de Holguín. CUBA. Doctor en Ciencias Técnicas, y docente de la propia institución. Líder del proyecto ELINF CUBA-Bélgica en el área de la Información, Bibliotecología y Tecnología Educativa. Con publicaciones en bases de datos como Scopus, Wos y Scielo. Entre sus áreas de investigación se encuentran las ciencias de la información, la dirección estratégica, gestión del conocimiento, tecnología educativa e inteligencia competitiva.

\section{Lic. Lourdes María Campins Marrero}

Directora del Centro de Inteligencia Competitiva Académica. Dirección de Información Científico Técnica. Universidad de Holguín. CUBA. Lic. Ciencias de la Información y docente de la propia institución. Miembro del proyecto ELINF Cuba-Bélgica. Entre sus áreas de investigación se encuentran las ciencias de la información, gestión del conocimiento, y las TIC.

\section{DrC. Ronal Tamayo Cuenca}

Metodólogo de la Vicerrectoría de Investigación y Postgrado. Universidad de Holguín. CUBA. Doctor en Ciencias Pedagógicas, docente y Metodólogo de Educación a Distancia de Postgrado en la Universidad de Holguín. Miembro del proyecto ELINF Cuba-Bélgica. Con más de 15 publicaciones. Sus áreas de investigación están enmarcadas en la tecnología educativa y educación a distancia. 